\title{
Ducumentation of Ethno Medicinal Plants of Gopalganj District of Bihar (India)
}

\author{
${ }^{1}$ Dr. Surendra kumar Prasad, ${ }^{2}$ Basant Narain Singh \\ ${ }^{I}$ Dept. of Botany Magadh Mahila College Patna University, Patna , India \\ ${ }^{2}$ Dept. of Botany, S.M.D. Degree college M.N. Jalalpur,Gopalganj, J.P. University,Chapra (India).
}

\begin{abstract}
Gopalganj, the small underdeveloped district in North- Western part of Bihar is bestowed with rich floral diversity and economically backward rural population covering $2033 \mathrm{Km}^{2}$ geographical area. The district is very close to the foothill of Himalayan ranges separated by river Gandak from west Champaran in east. The Present communication deals with the documentation of 221 Ethno Medicinal species out of 79 families, mostly from Angiosperms, a few from Gymnosperms and the few from Pteridophytes in alphabetical order. All these Ethno medicinal Plants are mentioned in this paper with their Botanical name, respective families, local name, life forms, Parts use and local uses.
\end{abstract}

Key words: Ethno Medicinal Plants, Gopalganj, rural population, diseases, district.

\section{Introduction}

The Gopalganj region is known for its diverse, unique, natural and socio- economical important flora. Gopalganj is the Small district in North- Western Bihar covering an area of $2033 \mathrm{Km}^{2}$.It lies between $26^{0} 39^{\prime}$ $\mathrm{N}$ Latitude and $83^{\circ} 54^{\prime}$ East Longitude. The altitude is 73 feet MSL. The soil of the district is thick alluvium intermittent deposited by river Gandak. The $\mathrm{pH}$ of the soil is mostly alkaline ranges from 7-8.The temperature ranges from $4^{\circ} \mathrm{C}$ in winter( December-January) and more than $44^{\circ} \mathrm{C}$ in hot Summer (May-June). The average annual rain fall is about $1170.90 \mathrm{~mm}$.

The district occupies one of the great archaic significance of places like Bhore, Thawe Durga Mandir and Daha river ; its reference appear in the epics like the Ramayan and Mahabharat. Now the Grah of Bhuishrwa in Bhore, the forest of Thawe near Thawe Durga Mandir and bank of river Daha is the best depository of Ethno Medicinal Plants. Inspite of these grasslands, the riverain banks of the river Gandak and their tributaries, the road side of National High way-28, the Saran main canal and its sub canals, the Railways sides are the rich Ethno Medicinal reservoir of the district.

Atharveda, the fourth and the last Vedas in Indian scripture, revealed the first Indian documented Scripture means the science of long life, appeared in the form of Ayurvedic medicine a system of traditional medicine native to the Indian sub- Continent. The most ancient and popular detailed texts on Ayurveda were composed by Charaka ( 600 BC ) and Shushruta ( first century ) provided information on medicinal Plants ( Devraj) 1985, Sharma and Goswami,(1992). In India medicinal needs of local Population mostly depends on plants as the natural sources of medication singh,(1998). The poor illiterate rural population and high class quality living persons equally believes in Ethno medicine.

\section{Materials And Methods}

The Present work was carried out for a frequent field trips in different seasons and different localities during the last few years. The Ethno medicinal information collected after frequent interview with rural old People, Gragiers, vaidyas, sadhu, house wifes etc. Proper information were also gathered from the Doctors Practicing in Ethno Medicines in the light of medicinal literature of Kritikar and Basu,(1935), Jja and Choudhary (1998), Annoymous (2006),Jain(2011).

\section{Results And Discussion}

The district has been blessed by nature with one of the richest vegetation of Ethno medicinal Plants from which the crude drugs can easily be produced . Rural population are economically, socially and educationally backward. The aim of the present study is to create local awareness regarding the importance, of Ethno medicinal plants for biodiversity conservation as well as community upliftment .

A total of 221 Ethno medicinal species belonging to 79 families ware recorded. Among Families Fabaceae (15 Species), Solanaceae (11 Species), Cucurbitaceae (10 Species) Asteraceae (9 Species), Caesalpineaceae (8 Species), Malvaceae, Moreceae and Apocynaceae (7 Species), Mimosaceae, Verbenaceae and Euphorbiaceae (6 Species), Rutaceae, Liliaceae, Apiaceae, Meliaceae and poaceae (5 Species), Amaranthaceae, chenopodiaceae, Myrtaceae, Zingiberaceae and Convolvulaceae (4 species ), Acanthaceae, 
Annonaceae, Areceaceae, Brassicaceae, Amaryllidaceae, Rubiaceae, oleaceae, Lamiaceae(2 speceis)and the remaining 40 families were monospecific (table-1).

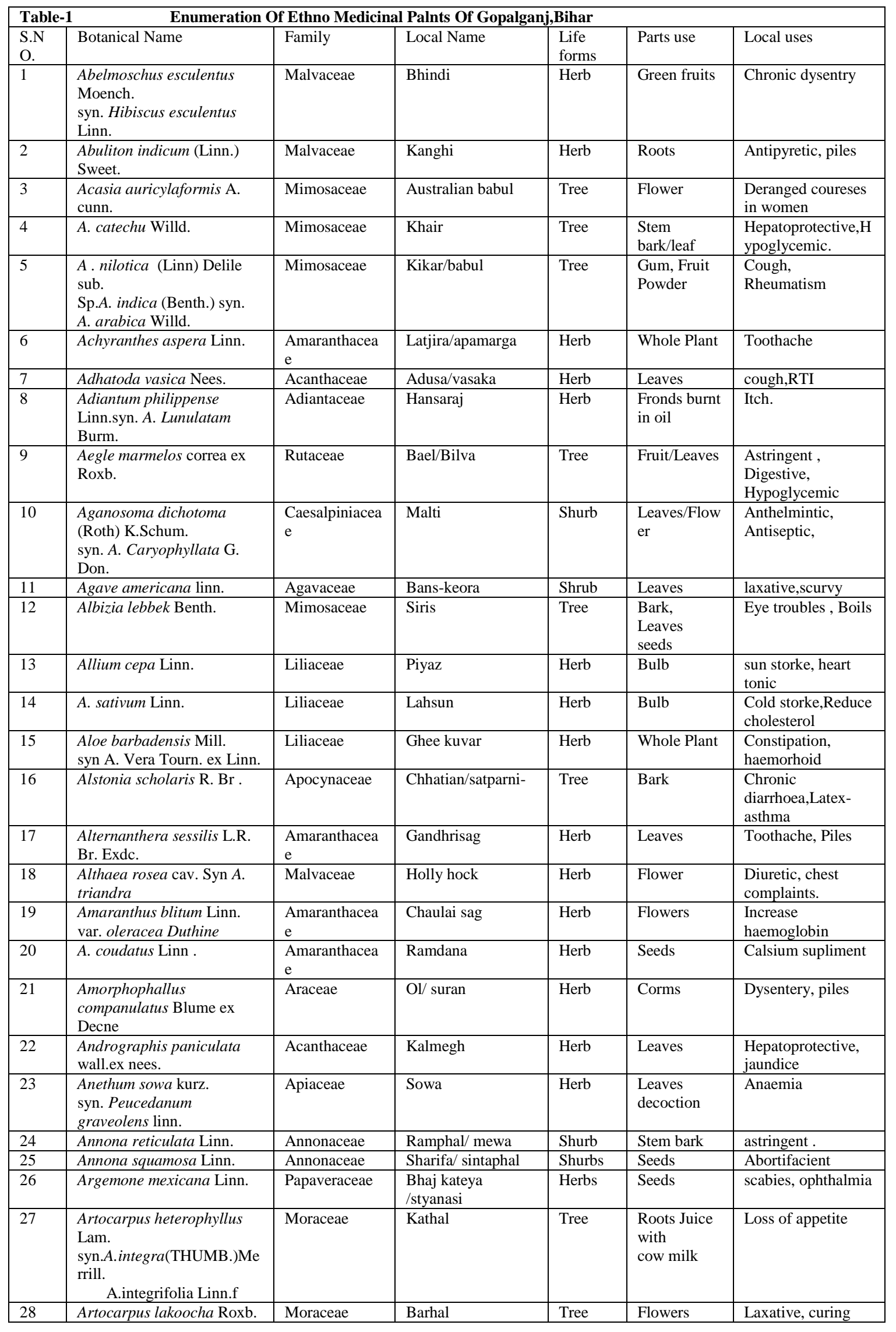


Ducumentation of Ethno Medicinal Plants of Gopalganj District of Bihar (India)

\begin{tabular}{|c|c|c|c|c|c|c|}
\hline & & & & & & liver \\
\hline 29 & Asparagus racemosus willd & Liliaceae & Satawar & Climber & Roots & $\begin{array}{l}\text { Galactogogue, } \\
\text { nervous, } \\
\text { Rheumatic } \\
\end{array}$ \\
\hline 30 & $\begin{array}{l}\text { Azadirachta indica A. juss . } \\
\text { syn.Melia azadirachta Linn. }\end{array}$ & Meliaceae & Nim & Tree & Whole plant & $\begin{array}{l}\text { Skin disease, blood } \\
\text { purifier }\end{array}$ \\
\hline 31 & Bambusa sp. & Poaceae & Banas & Shrubs & $\begin{array}{l}\text { Green Stem } \\
\text { Peel }\end{array}$ & $\begin{array}{l}\text { Dysentery, } \\
\text { diarrhoea }\end{array}$ \\
\hline 32 & $\begin{array}{l}\text { Basella alba Linn. Syn. } B \text {. } \\
\text { rubra Linn. }\end{array}$ & Basellaceae & Poi & Climber & Leaves & $\begin{array}{l}\text { Rejuvenative, } \\
\text { Retrigerant. }\end{array}$ \\
\hline 33 & Bauhinia variegata linn. & $\begin{array}{l}\text { Caesalpiniacea } \\
\text { e }\end{array}$ & Kachnar & Shrubs & Stem bark & Tonic in feaver \\
\hline 34 & $\begin{array}{l}\text { Biota orientalis Ende. } \\
\text { syn.Thuja orientalis Linn. }\end{array}$ & Cupressaceae & Morpankhi & Shrubs & $\begin{array}{l}\text { Flat Green } \\
\text { branch }\end{array}$ & Fever, cough \\
\hline 35 & $\begin{array}{l}\text { Beta vulgaris Linn var.rapa } \\
\text { Dum. }\end{array}$ & $\begin{array}{l}\text { Chenopodiace } \\
\text { ae }\end{array}$ & Chukandar & Herbs & Roots & Rheumatoid \\
\hline 36 & $\begin{array}{l}\text { Boerhaavia diffusa linn. } \\
\text { Syn. B. repns Linn. }\end{array}$ & Nyctaginaceae & Punarnava & Herbs & Whole plant & Diuretic, Jaundice \\
\hline 37 & $\begin{array}{l}\text { Bombax ceba Linn. } \\
\text { syn. Salmalia } \\
\text { malabarica(DC) Schott \& } \\
\text { Endl.B.malabaricum(DC.) }\end{array}$ & Bombaceae & Semul & Tree & $\begin{array}{l}\text { Fruits/Stem } \\
\text { bark }\end{array}$ & $\begin{array}{l}\text { Stimulant,Expector } \\
\text { ant, diuretic, } \\
\text { Demulcent,tonic,st } \\
\text { yptic. }\end{array}$ \\
\hline 38 & $\begin{array}{l}\text { Borassus } \\
\text { flobelliferaLinn.syn. } B . \\
\text { flabelliformis } \text { Roxb. }\end{array}$ & Arecaceae & Tadkul/Tar & Tree & $\begin{array}{l}\text { Toddy/palm } \\
\text { Juice }\end{array}$ & Constipation. \\
\hline 39 & Brassica compestris Linn. & Brassicaceae & Sarson & Herbs & $\begin{array}{l}\text { Oil with } \\
\text { comphore }\end{array}$ & $\begin{array}{l}\text { muscular } \\
\text { Rheumatoid, }\end{array}$ \\
\hline 40 & $\begin{array}{l}\text { Bryophyllum pinnatum } \\
\text { (Lam.) Kuntz. } \\
\text { syn.B.calycinum Salisb. }\end{array}$ & Crassulaceae & $\begin{array}{l}\text { Patherchur/Pasanbh } \\
\text { edi }\end{array}$ & Herbs & Leaves & Haemoptysis, stone \\
\hline 41 & $\begin{array}{l}\text { Butea monosperma (Lam.) } \\
\text { Kurz } \\
\text { syn } B . \text { frondosa koenig ex } \\
\text { Roxb. }\end{array}$ & Fabaceae & Palas /Dhak & Tree & Stem bark & $\begin{array}{l}\text { Piles, tumour, } \\
\text { M.C.disorders }\end{array}$ \\
\hline 42 & $\begin{array}{l}\text { Cajanus cajan (Linn.) Mill } \\
\text { sp } \\
\text { syn. C.Indicus spreng. }\end{array}$ & Fabaceae & Arhar & Shurbs & Leaves & Jaundice \\
\hline 43 & Callistemon lancelatus DC. & Myrtaceae & Bottle brush & Shurbs & Leaves & $\begin{array}{l}\text { Poultice of leaves } \\
\text { applied on } \\
\text { vaginites. }\end{array}$ \\
\hline 44 & $\begin{array}{l}\text { Colotropis gigantea (Linn) } \\
\text { R.Br.ex.Ait. }\end{array}$ & $\begin{array}{l}\text { Asclepiadacea } \\
\mathrm{e}\end{array}$ & Ak & Shurbs & Leaves & $\begin{array}{l}\text { Swelling pain } \\
\text { disease }\end{array}$ \\
\hline 45 & C.procera (Ait.) R. Br. & $\begin{array}{l}\text { Asclepiadacea } \\
\mathrm{e}\end{array}$ & Mandar & Shurbs & Root bark & leprosy \\
\hline 46 & Cannabis sativa Linn. & Cannabinaceae & Bhang & Shurbs & Leaves & $\begin{array}{l}\text { Sedative, } \\
\text { appetising }\end{array}$ \\
\hline 47 & Copsicum annum Linn. & Solanaceae & Lalmirch & Herbs & Fruits & $\begin{array}{l}\text { Carminative, } \\
\text { rheumatism }\end{array}$ \\
\hline 48 & Carica papaya Linn & Caricaceae & Papeeta & Shurbs & Roots Juice & Stone \\
\hline 49 & Carissa carandas Linn. & Apocynaceae & Karaunda & Shurbs & $\begin{array}{l}\text { Leavers } \\
\text { decoction }\end{array}$ & Remittent Finvers \\
\hline 50 & Cassia fistula Linn & $\begin{array}{l}\text { Caesalpiniacea } \\
\text { e }\end{array}$ & Amaltas & Tree & Fruits & Purgative, laxative \\
\hline 51 & C. occidentalis Linn. & $\begin{array}{l}\text { Caesalpiniacea } \\
\mathrm{e}\end{array}$ & Kasmard/kasaundi & Herb & $\begin{array}{l}\text { Leavers } \\
\text { seeds }\end{array}$ & $\begin{array}{l}\text { Purgative, Skin } \\
\text { trouble }\end{array}$ \\
\hline 52 & C. tora Linn. & $\begin{array}{l}\text { Caesalpiniacea } \\
\text { e }\end{array}$ & $\begin{array}{l}\text { Chakamrd/chakund } \\
\text { a }\end{array}$ & Herb & Leavers & Purgative, laxative \\
\hline 53 & Carthamus tinctorius Linn. & Asteraceae & Kusum & Herbs & Flower, & Iaxative, jaundice \\
\hline 54 & $\begin{array}{l}\text { Cedrela toona } \text { Roxb .syn. } \\
\text { Toona eiliata M. Roem. }\end{array}$ & Meliaceae & Tunt & Tree & Bark & $\begin{array}{l}\text { Chronic dysentry } \\
\text { ulcer }\end{array}$ \\
\hline 55 & $\begin{array}{l}\text { Centella asiatica } \\
\text { (Linn).Urban. } \\
\text { syn . Hydrocotyle asiatica } \\
\text { Linn. }\end{array}$ & Apiaceae & $\begin{array}{l}\text { Bramhi / } \\
\text { Mandukparni }\end{array}$ & Herb & Leaves & $\begin{array}{l}\text { Brain tonic, } \\
\text { Diuretic }\end{array}$ \\
\hline 56 & $\begin{array}{l}\text { Chenopodium album Linn. } \\
\text { Lamb's }\end{array}$ & $\begin{array}{l}\text { Chenopodiace } \\
\text { ae }\end{array}$ & Bathua sag & Herb & whole plant & Anti anaemia \\
\hline 57 & Cicer arientinum Linn. & Fabaceae & Chana /But & Herb & Seeds & Heart disease \\
\hline 58 & $\begin{array}{l}\text { Cissus quadrangularis } \\
\text { Linn.Cissus quadrngularis } \\
\text { Linn.syn.Vitis } \\
\text { quadrngularis wall. }\end{array}$ & Vitaceae & Harzorwa & Climber & Leaves & Bone healing \\
\hline 59 & $\begin{array}{l}\text { Citrus allrantifolia(christm } \\
\text { ) Swingle }\end{array}$ & Rutaceae & Kaghzinimbu & Shurbs & Fruits & Stomachic, \\
\hline
\end{tabular}


Ducumentation of Ethno Medicinal Plants of Gopalganj District of Bihar (India)

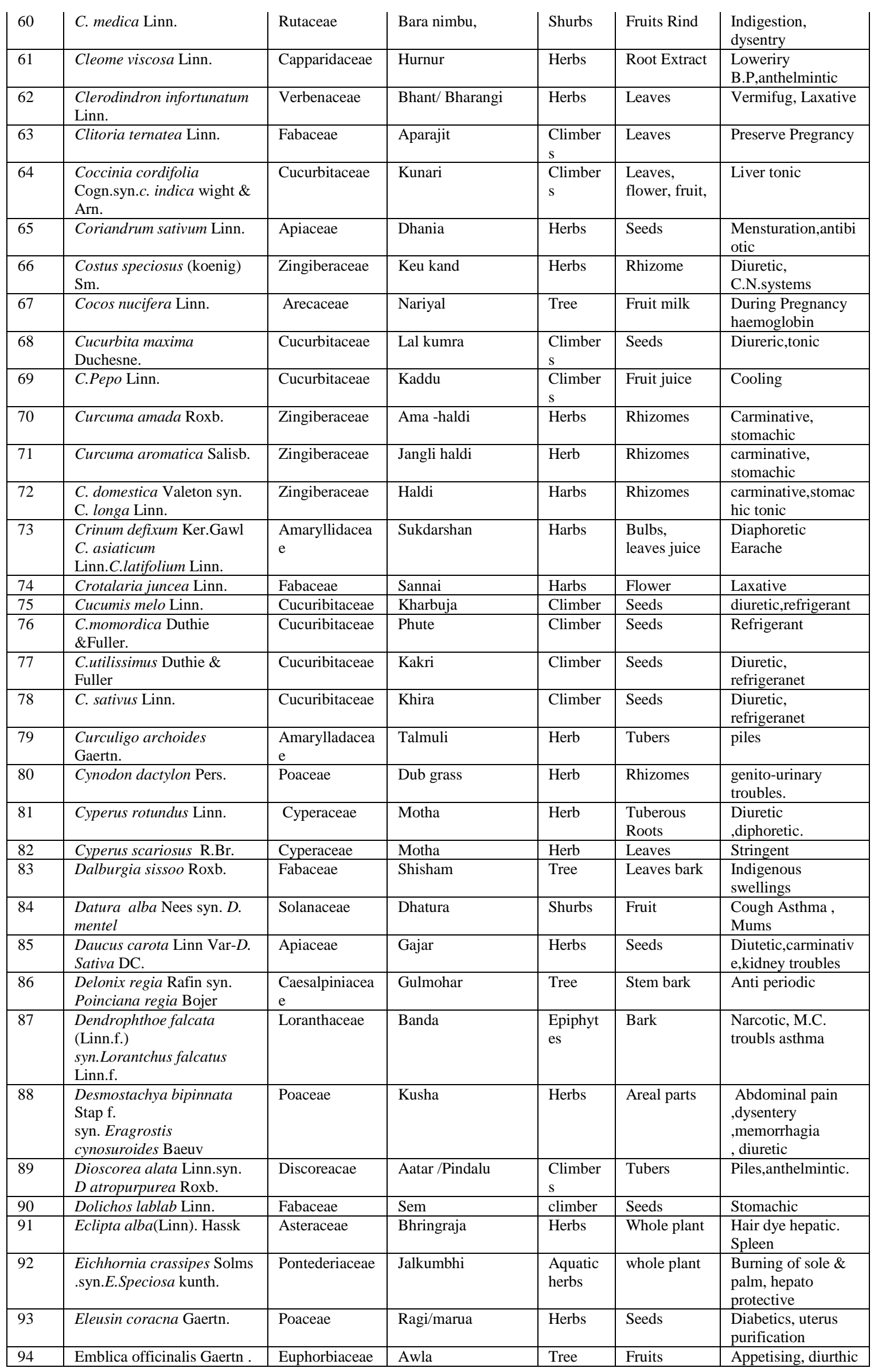




\begin{tabular}{|c|c|c|c|c|c|c|}
\hline & $\begin{array}{l}\text { syn.phyllanthus emblica } \\
\text { Linn. }\end{array}$ & & & & & laxative \\
\hline 95 & $\begin{array}{l}\text { Erythrina indica Lam. Syn. } \\
\text { E. variegata Linn. }\end{array}$ & Fabaceae & Farhad/Mandar & Tree & Bark & $\begin{array}{l}\text { Leprosy, Feaver } \\
\text {,Blood dysentry }\end{array}$ \\
\hline 96 & Equisetum debile Roxb. & Equisetaceae & - & Herb & whole Plant & $\begin{array}{l}\text { Gonorrhoea, } \\
\text { diuretic }\end{array}$ \\
\hline 97 & $\begin{array}{l}\text { Eucalyptus treticornis } \\
\text { SM.syn. E. umbellata } \\
\text { Domin. }\end{array}$ & Myrtaceae & Safeda & Tree & $\begin{array}{l}\text { Oil form } \\
\text { leaves }\end{array}$ & Cold \\
\hline 98 & $\begin{array}{l}\text { Euphorbia hirta } \text { Linn } \\
\text {.syn.E.pilulifera } \text { Acuct. }\end{array}$ & Euphorbiaceae & Dudhi & Herb & Leaves & $\begin{array}{l}\text { Preserve } \\
\text { pregnancy, Milk } \\
\text { inhancer }\end{array}$ \\
\hline 99 & E. neriifolia Linn. & Euphorbiaceae & Sehund & Shurbs & Leaves latex & $\begin{array}{l}\text { Purgative, } \\
\text { expectorant }\end{array}$ \\
\hline 100 & Ficus bengalensis Linn. & Moraceae & Bargad & Tree & $\begin{array}{l}\text { Leaves,Prop } \\
\text { root }\end{array}$ & $\begin{array}{l}\text { Diabetes,Pain } \\
\text { reumatism }\end{array}$ \\
\hline 101 & F.benjamina Linn. & Moraceae & Pakar & Tree & $\begin{array}{l}\text { Leaves, } \\
\text { dicoction }\end{array}$ & Ulcers. \\
\hline 102 & $\begin{array}{l}\text { F.glomerata } \\
\text { Roxb.syn.F.Racemosa Linn. }\end{array}$ & Moraceae & Gular & Tree & Root & $\begin{array}{l}\text { Diarrhoea, diabets } \\
\text { Laxative }\end{array}$ \\
\hline 103 & F.religiosa Linn. & Moraceae & Pipal/Ashvatha & Tree & Fruits & Laxative \\
\hline 104 & Gamelina Arborea Roxb. & Verbenacae & Gambhar & Tree & whole Plant & $\begin{array}{l}\text { Abortion and } \\
\text { Prolapses piles }\end{array}$ \\
\hline 105 & $\begin{array}{l}\text { Gardenia Jasminoides } \\
\text { Ellis.syn. G. florida Linn. } \\
\text { G. augusta merrill. }\end{array}$ & Rubiaceae & Gandhraj & Shurb & Root & $\begin{array}{l}\text { Dyspepsia.and } \\
\text { nervous disorders. }\end{array}$ \\
\hline 106 & $\begin{array}{l}\text { Gossypium arboreum Linn. } \\
\text { syn.G.nan king }\end{array}$ & Malvaceae & Patua & Herbs & $\begin{array}{l}\text { Root } \\
\text { Tincture }\end{array}$ & $\begin{array}{l}\text { Prevent frequent } \\
\text { abortion \& } \\
\text { prolapses }\end{array}$ \\
\hline 107 & Helianthus annus Linn. & Asteraceae & Surajmukhi & Herbs & Seeds & $\begin{array}{l}\text { Anthelmintic, } \\
\text { Rubefacient }\end{array}$ \\
\hline 108 & $\begin{array}{l}\text { Heliotropicum indicum } \\
\text { Linn. }\end{array}$ & Boraginaceae & Hathisund & Herb & whole Plant & $\begin{array}{l}\text { Diuretic, ulcer, } \\
\text { Feaver }\end{array}$ \\
\hline 109 & $\begin{array}{l}\text { Hibiscus rosa- sinensis } \\
\text { Linn. }\end{array}$ & Malvaceae & Gurhal & Shurbs & Flower & Abortifacient, \\
\hline 110 & Hibiscus cannabinus Linn. & Malvaceae & Patsan & Herbs & Leaves & Purgative \\
\hline 111 & $\begin{array}{l}\text { Hygrophila angustifolia } \\
\text { Auct. } \\
\text { syn. H salicifolia Nees. }\end{array}$ & Acanthaceae & Talmakhana & Herbs & whole Plant & $\begin{array}{l}\text { Jaundice, } \\
\text { urinogenital system }\end{array}$ \\
\hline 112 & Impatiens balsamia Linn. & $\begin{array}{l}\text { Balsa } \\
\text { minaceae }\end{array}$ & Gul-mehindi & Herbs & Flowers & $\begin{array}{l}\text { Lumbago } \\
\text { intercostal } \\
\text { neuralgia } \\
\end{array}$ \\
\hline 113 & $\begin{array}{l}\text { Ipomoea aquotica Forsk } \\
\text {.Syn. I. reptans } \text { Poir }\end{array}$ & $\begin{array}{l}\text { Convolvulacea } \\
\mathrm{e}\end{array}$ & Kalmisag & Creeper & whole Plant & $\begin{array}{l}\text { Wholesome for } \\
\text { women, ematic }\end{array}$ \\
\hline 114 & I. batatas .(Linn). Lam. & $\begin{array}{l}\text { Convolvulacea } \\
\text { e }\end{array}$ & $\begin{array}{l}\text { Maith alu } \\
\text { /shakarkaknd }\end{array}$ & Climber & $\begin{array}{l}\text { (tuberow } \\
\text { root), } \\
\text { Leaves }\end{array}$ & Laxative \\
\hline 115 & $\begin{array}{l}\text { I. cairica (Linn.) Sweet syn. } \\
\text { I. palmat Forsk. }\end{array}$ & $\begin{array}{l}\text { Convolvulacea } \\
\mathrm{e}\end{array}$ & Behaya & Shurb & Seeds & Pugative \\
\hline 116 & I. digitata Linn. & $\begin{array}{l}\text { Convolvulacea } \\
\mathrm{e}\end{array}$ & Vidani kand & Climber & $\begin{array}{l}\text { Root } \\
\text {,Leaves }\end{array}$ & Bone healing \\
\hline 117 & Ixora coccinia Linn. & Rubiaceae & Rookmini & Shurbs & Roots & Stomachic, sedative \\
\hline 118 & $\begin{array}{l}\text { Jasminum arborescens } \\
\text { Roxb. } \\
\text { syn.J.roxburghianum Wall. }\end{array}$ & Oleaceae & Bela /chameli & Shurbs & Leaves & $\begin{array}{l}\text { Bronchial } \\
\text { obstruction } \\
\text { stamachic, tonic }\end{array}$ \\
\hline 119 & J. grandiflorum Linn. & Oleacae & Chameli & Climber & Leaves dried & $\begin{array}{l}\text { Pultices for } \\
\text { indolent ulcer. }\end{array}$ \\
\hline 120 & $\begin{array}{l}\text { Jatropha glandulitera } \\
\text { Roxb. }\end{array}$ & Euphorbiaceae & Janglive rendi & Shurbs & Seeds & $\begin{array}{l}\text { Purgative, } \\
\text { rheumatism, }\end{array}$ \\
\hline 121 & Kochia indica wight. & $\begin{array}{l}\text { Chenopodiace } \\
\text { ae }\end{array}$ & Kochia & Herb & Leaves & Cardiac stimulant, \\
\hline 122 & Lactuca sativa Linn. & Asteraceae & Salad Patta & Herb & Leaves & $\begin{array}{l}\text { Hypnotic in } \\
\text { bronchits ,Asthma. }\end{array}$ \\
\hline 123 & $\begin{array}{l}\text { Lantana camara Linn.syn. } \\
\text { L. aculeata Linn. }\end{array}$ & Verbenaceae & Lantana & Herb & Leaves & $\begin{array}{l}\text { Chronic feaver, } \\
\text { RTI, }\end{array}$ \\
\hline 124 & $\begin{array}{l}\text { Launaea asplenifolia Hook } \\
\text {.f. }\end{array}$ & Asteraceae & Titlia & Herb & Roots & $\begin{array}{l}\text { Constituent of } \\
\text { galacta gogue drugs }\end{array}$ \\
\hline 125 & $\begin{array}{l}\text { Lawsonia inermis Linn. } \\
\text { Syn.L.alba Lam. }\end{array}$ & Lythraceae & Mehandi & Shurbs & Leaves & $\begin{array}{l}\text { Refregerant to } \\
\text { mind,palms }\end{array}$ \\
\hline 126 & Letipidium sativum Linn. & Brassicaceae & Chansur & Herb & Leaves & Liver tonic \\
\hline 127 & Leucas aspera Spreng. & Lamiaceae & Gumi & Herb & Flower & $\begin{array}{l}\text { Jaundice, spleene } \\
\text { asthma }\end{array}$ \\
\hline
\end{tabular}


Ducumentation of Ethno Medicinal Plants of Gopalganj District of Bihar (India)

\begin{tabular}{|c|c|c|c|c|c|c|}
\hline 128 & L. cephalotes Spreng. & Lamiaceae & $\begin{array}{l}\text { Dronpushapi } \\
\text { /Guma }\end{array}$ & Herb & Flower & $\begin{array}{l}\text { Skin disease } \\
\text { Jaundice }\end{array}$ \\
\hline 129 & Linum usitatissimum Linn. & Linaceae & Tisi/Alsi & Herb & $\begin{array}{l}\text { Seeds with } \\
\text { honey }\end{array}$ & Cough \\
\hline 130 & $\begin{array}{l}\text { Luffa acutangula (Linn.) } \\
\text { Roxb. }\end{array}$ & Cucurbitaceae & Sataputia troi & Climber & Pultice & $\begin{array}{l}\text { Hemorrhoids, } \\
\text { Leprosy }\end{array}$ \\
\hline 131 & $\begin{array}{l}\text { Lycopersicon esculentum } \\
\text { Mill. }\end{array}$ & Solanaceae & Tamatar & Herb & Fruit & Blood purifier \\
\hline 132 & $\begin{array}{l}\text { Madhuca indica J.F.Gmel } \\
\text { syn.M.Latifolia } \text { Macbr. } \\
\text { Bassia latifolia } \text { Roxb. }\end{array}$ & Sapotaceae & Mahua & Tree & Seed soil & $\begin{array}{l}\text { Rheumatism, } \\
\text { Laxative Piles }\end{array}$ \\
\hline 133 & Mangifera indica Linn. & Anacardiaceae & Aam & Tree & $\begin{array}{l}\text { Tendre } \\
\text { leaves }\end{array}$ & Diabetes \\
\hline 134 & Melia azedarach Linn. & Meliaceae & Bakain & Tree & $\begin{array}{l}\text { Seeds/Leaf } \\
\text { Juice }\end{array}$ & $\begin{array}{l}\text { Asthma, Bark- } \\
\text { uterine } \\
\text { haemorrhege } \\
\text { anthelmintic, } \\
\text { diuretic }\end{array}$ \\
\hline 135 & Mentha arvensis Linn. & Lamiaceae & Pudina & Herb & $\begin{array}{l}\text { Fruits/ } \\
\text { Leaves }\end{array}$ & $\begin{array}{l}\text { Tonic. Stimulant, } \\
\text { carminative }\end{array}$ \\
\hline 136 & Michelia champaca Linn. & Magnoliaceae & Champa & Harbs & Flower & $\begin{array}{l}\text { Carminative, } \\
\text { dyspepsia. }\end{array}$ \\
\hline 137 & Mimosa pudica Linn. & Mimosaceae & Lajwanti & Herbs & Leaves & $\begin{array}{l}\text { Piles, nausea, } \\
\text { Feaver }\end{array}$ \\
\hline 138 & Mimusops elengi Linn. & Sapotaceae & Maulsari & Tree & Stem bark & Uterin purification \\
\hline 139 & Mirabilis Jalapa Linn. & Nyctaginaceae & Gulabash & Herb & Leaves Juice & Blood purifier \\
\hline 140 & $\begin{array}{l}\text { Mitragyna parvifolia } \\
\text { (Roxb.) korth. } \\
\text { syn. Stephegyne parvifolia } \\
\text { Korth. }\end{array}$ & Rubiaceae & Kadamb & Tree & Stem, fruits & $\begin{array}{l}\text { Vaginitis, } \\
\text { spermopoetic. }\end{array}$ \\
\hline 141 & Momordica charantia Linn. & Cucarbitaceae & Karela & Climber & Fruits & Hypoglycemic. \\
\hline 142 & $\begin{array}{l}\text { Maringa olifera lam. } \\
\text { syn.M.pterygosperma } \\
\text { Gaertn. }\end{array}$ & Moringaceae & Sainjana/Shajna & Tree & $\begin{array}{l}\text { Leaves, } \\
\text { Fruits }\end{array}$ & $\begin{array}{l}\text { Cardio tonic } \\
\text { diuretic }\end{array}$ \\
\hline 143 & Morus alba Linn. & Moraceae & Shahtut & Shurbs & Fruits & $\begin{array}{l}\text { Refrigerant, } \\
\text { purgative. }\end{array}$ \\
\hline 144 & $\begin{array}{l}\text { Mucana prurita } \\
\text { Hook.syn.M.pruriens } \\
\text { Baker. }\end{array}$ & Fabaceae & Kaunch/ kwachh. & Climber & Pods roots & $\begin{array}{l}\text { Hypoglycemic, } \\
\text { parkinson disease. }\end{array}$ \\
\hline 145 & $\begin{array}{l}\text { Murraya koenigii } \\
\text { (Linn.)Spreng. }\end{array}$ & Rutaceae & Kurry patta & Shurbs & $\begin{array}{l}\text { Root , } \\
\text { barkes }\end{array}$ & $\begin{array}{l}\text { Carminative, } \\
\text { diarrhoea }\end{array}$ \\
\hline 146 & $\begin{array}{l}\text { M. paniculata (linn). Jack } \\
\text { syn. M.exotica Linn. }\end{array}$ & Rutaceae & Kamini & Shurbs & Leaves & Diarrhoea, dysentry \\
\hline 147 & $\begin{array}{l}\text { Musa paradisica } \\
\text { Linn.syn.M.sapientum Linn. }\end{array}$ & Musaceae & Kela & Herb & Stem juice & $\begin{array}{l}\text { dysentry.Laxative, } \mathrm{h} \\
\text { ypertension. }\end{array}$ \\
\hline 148 & $\begin{array}{l}\text { Nelumbo nucifere Gaertn. } \\
\text { syn.Nelumbium nelumbo } \\
\text { Druce.N.speciosum Willd. }\end{array}$ & Nymphyaceae & Kamal & Herb & Rhizomes & $\begin{array}{l}\text { diarrhoea,dysentery } \\
\text { (children) }\end{array}$ \\
\hline 149 & $\begin{array}{l}\text { Nerium Indicum Mills. } \\
\text { syn.N.odorum Soland. }\end{array}$ & Apocynaceae & Kanev/karber & Shurbs & Leaves & $\begin{array}{l}\text { cardio tonic skin } \\
\text { disorders. }\end{array}$ \\
\hline 150 & $\begin{array}{l}\text { Nicotiana plumbaginifolia } \\
\text { viv }\end{array}$ & Solanaceae & Bantamaku & Herb & Leaves & $\begin{array}{l}\text { painful tumours, } \\
\text { gonorrhoea. }\end{array}$ \\
\hline 151 & N. tabacum Linn. & Solanaceae & Tambaku/surti & Herb & Leaves & $\begin{array}{l}\text { sedative, } \\
\text { antispasmodic, } \\
\text { gastric. }\end{array}$ \\
\hline 152 & $\begin{array}{l}\text { Nyctanthes arbortristis } \\
\text { Linn. }\end{array}$ & Oleacae & Harsinghar & Shurbs & Leaves & $\begin{array}{l}\text { feaver,antibilious,e } \\
\text { xpectorant } \\
\text { raeumatism.sciatica }\end{array}$ \\
\hline 153 & Nymphaea alba Linn. & Nymphaeaceae & water lily & Herb & Rhizomes & $\begin{array}{l}\text { dicoction in } \\
\text { diarrhoea. }\end{array}$ \\
\hline 154 & Ocimum basilicum Linn. & Lamiaceae & Tulsi & Herb & whole Plant & $\begin{array}{l}\text { Expectorant, } \\
\text { carminative, }\end{array}$ \\
\hline 155 & O.sanctum Linn. & Lamiaceae & Tuls /Brinda & Herb & whole Plant & $\begin{array}{l}\text { Antibacterial, } \\
\text { Expectorant. }\end{array}$ \\
\hline 156 & $\begin{array}{l}\text { Orobanche indica } \\
\text { Buch.Ham ex Roxb. }\end{array}$ & Orobanchaceae & Thokra/Gadhaura & Parasite & whole Plant & Diarrhoea \\
\hline 157 & Opuntia dillenii Haw. & Cactaceae & Nagphani & Herb & Baked fruits & Whooplng cough. \\
\hline 158 & Oxalis corniculata Linn. & Oxalidaceae & Khhatibuti & Herbs & Leaves & $\begin{array}{l}\text { Dyspepsia,piles,ana } \\
\text { emia, }\end{array}$ \\
\hline 159 & $\begin{array}{l}\text { Pandanus andamanen Sium } \\
\text { Kurz. } \\
\text { P.fascicularisLam. }\end{array}$ & Pandanaceae & Keore & Sburbs & Flower juice & Cardio tonic \\
\hline 160 & Parthenium hysterophorum & Asteraceae & Gajarghars & Herb & whole Plant & Febrifuse, \\
\hline
\end{tabular}


Ducumentation of Ethno Medicinal Plants of Gopalganj District of Bihar (India)

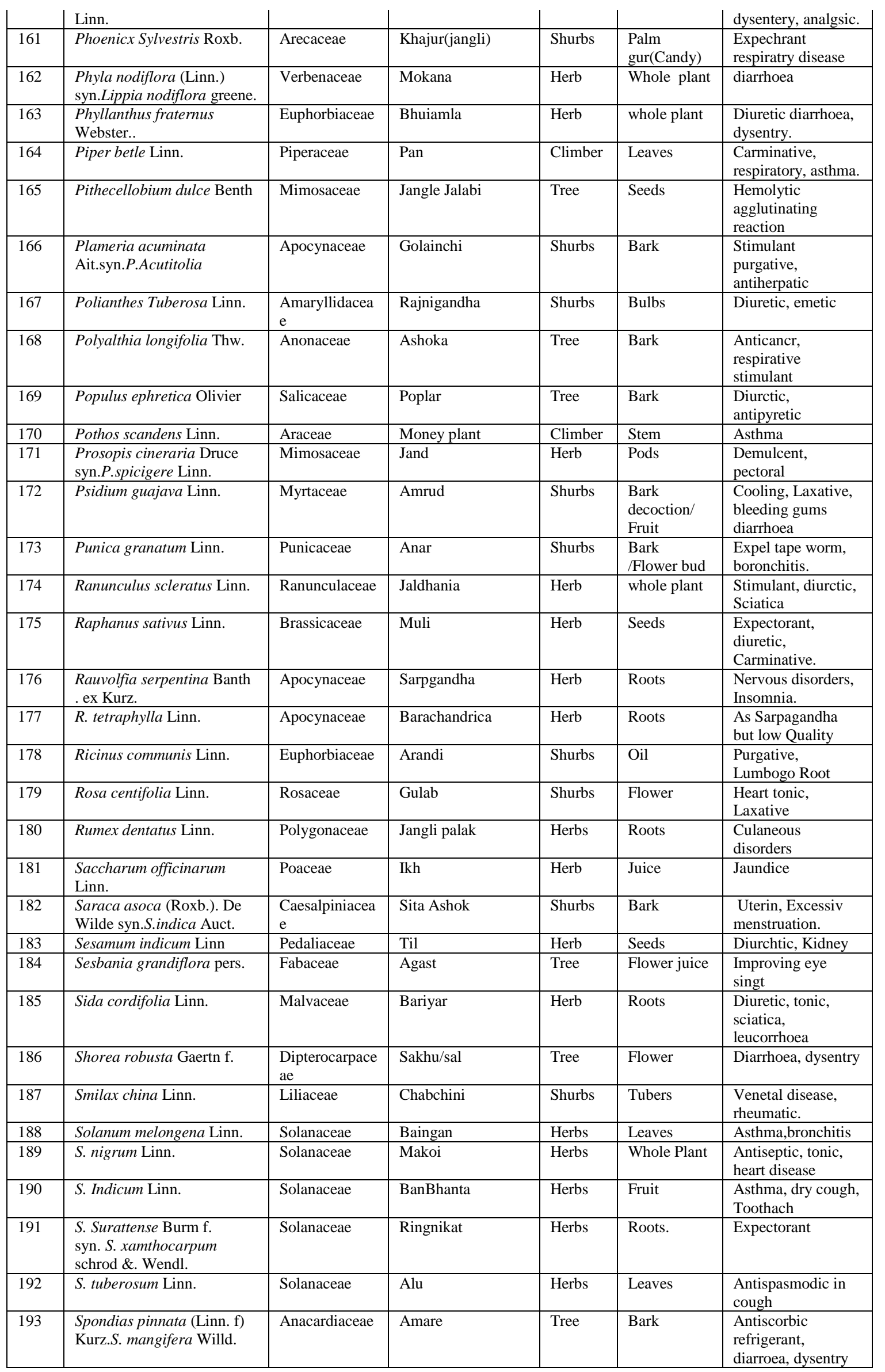


Ducumentation of Ethno Medicinal Plants of Gopalganj District of Bihar (India)

\begin{tabular}{|c|c|c|c|c|c|c|}
\hline 194 & Spinacia odereca Linn. & $\begin{array}{l}\text { Chenopodiace } \\
\text { ae }\end{array}$ & Palak & Herb & $\begin{array}{l}\text { Leaves/Fruit } \\
\mathrm{s}\end{array}$ & $\begin{array}{l}\text { Antibacterial,diuret } \\
\text { ic,Feaver, }\end{array}$ \\
\hline 195 & Stevia rebadiana Burtoni & Asteraceae & Madhurguni & Herb & Leaves & $\begin{array}{l}\text { Sweetner for } \\
\text { diabets. }\end{array}$ \\
\hline 196 & Swietenia macrophylla King & Meliaceae & Mahogani & Tree & Bark & jetty piles \\
\hline 197 & S. mahogoni Jacq. & Meliaceae & $\begin{array}{l}\text { Mahogani(Large } \\
\text { leaf) }\end{array}$ & Tree & Bark & Antipyctic. Tonic. \\
\hline 198 & $\begin{array}{l}\text { Syzygium cuminii (Linn.) } \\
\text { Skeels. } \\
\text { syn.Euginia jambolana } \\
\text { Lam. }\end{array}$ & Myrtaceae & Jamun & Tree & Fruit, seed & Hypoglycemic. \\
\hline 199 & Tagetes patula Linn. & Asterceae & Genda & Herbs & $\begin{array}{l}\text { Leaves, } \\
\text { Fruit }\end{array}$ & Blood coagulant \\
\hline 200 & Tamarindus Indica Linn & $\begin{array}{l}\text { Caesalpiniacea } \\
\mathrm{e}\end{array}$ & Imli & Tree & Fruits & $\begin{array}{l}\text { Carminative, } \\
\text { laxative, }\end{array}$ \\
\hline 201 & $\begin{array}{l}\text { Tamarix troupii Hole. syn. } \\
\text { T. golliea Auet. }\end{array}$ & Tamricaceae & Jhav & Shurb & Leaves & Diarrhoea dysentry \\
\hline 202 & Tectona grandis Linn $\mathrm{f}$. & Verbenaceae & Sagun & Tree & $\begin{array}{l}\text { Kernels } \\
\text { flower }\end{array}$ & Scabies, bronchitis \\
\hline 203 & Tephrosia purpurea Pers. & Fabaceae & Sarphonka & Herb. & Whole plant & $\begin{array}{l}\text { Tonic, laxative, } \\
\text { diuretc, }\end{array}$ \\
\hline 204 & $\begin{array}{l}\text { Terminalia arjuna (Roxb) } \\
\text { Wight \& Arn. }\end{array}$ & Combretaceae & Arjuna & Tree & Bark & $\begin{array}{l}\text { Symptomatic } \\
\text { hypertension , heat } \\
\text { disease }\end{array}$ \\
\hline 205 & $\begin{array}{l}\text { Thevetia peruviana (Pers) } \\
\text { Merrill Linn. } \\
\text { syn.T.neriifolia juss. ex } \\
\text { Sterd. }\end{array}$ & Apocynaceae & Pila kaner & Shurb & Leaves & Purgative, emetic. \\
\hline 206 & $\begin{array}{l}\text { Tinospora cordifolia } \\
\text { (Willd.) Miers ex Hook f. \& } \\
\text { Thoms }\end{array}$ & $\begin{array}{l}\text { Menispermace } \\
\text { ae }\end{array}$ & Giloe/Amrita & Climber & Leaves/Stem & $\begin{array}{l}\text { Fever, antiyretic, } \\
\text { leprosy }\end{array}$ \\
\hline 207 & $\begin{array}{l}\text { Trapa natens } \\
\text { Linn.syn.T.bispinosa Roxb. }\end{array}$ & Trapaceae & Singhara & $\begin{array}{l}\text { Aquatic } \\
\text { Herb }\end{array}$ & Fruit & $\begin{array}{l}\text { Abdominal } \\
\text { disorders }\end{array}$ \\
\hline 208 & Tribulus terrestris Linn. & $\begin{array}{l}\text { Zygophyllacea } \\
\text { e }\end{array}$ & Gokhru & Herb & Fruit & $\begin{array}{l}\text { Tonic., diuretic, } \\
\text { stomachic, }\end{array}$ \\
\hline 209 & $\begin{array}{l}\text { Trigonella foenum graecum } \\
\text { Linn. }\end{array}$ & Fabaceae & Methi & Herb & Leaves fruits & $\begin{array}{l}\text { Carminative, tonic, } \\
\text { hypoglycemic }\end{array}$ \\
\hline 210 & $\begin{array}{l}\text { Trachyspermum ammi } \\
\text { (Linn) Sprague . } \\
\text { Syn.T.copiticum Link. }\end{array}$ & Apiaceae & Ajwain & Herb & Fruit & $\begin{array}{l}\text { Antispasmodic, } \\
\text { carminative, }\end{array}$ \\
\hline 211 & Trichosanthes dioca Roxb. & Cucurbitaceae & Parwall & Climber & Fruits & $\begin{array}{l}\text { Laxative, cancer, } \\
\text { hemogglutinating } \\
\text { activhy. }\end{array}$ \\
\hline 212 & $\begin{array}{l}\text { Typha angustata Bory \& } \\
\text { Chaub. }\end{array}$ & Typhaceae & Patera & Herb & Rhizome & Diuretic. \\
\hline 213 & Vicia faba Linn. & Fabaceae & Bakla & Herb & Bean & $\begin{array}{l}\text { Jaundice, } \\
\text { hemolaytic } \\
\text { anaemia }\end{array}$ \\
\hline 214 & $\begin{array}{l}\text { Vigna mungo (Linn.) } \\
\text { Hepper syn. Phaselus } \\
\text { radiatus Roxb. }\end{array}$ & Fabaceae & Urd & Herb & Pulse & $\begin{array}{l}\text { Rhematism, } \\
\text { nervous, hepatic. }\end{array}$ \\
\hline 215 & $\begin{array}{l}\text { V. radiata (Linn) Wilczek . } \\
\text { syn. Phaseolus mungo Auet }\end{array}$ & Fabaceae & Mung & Herb & Seeds & $\begin{array}{l}\text { Polynuritis, } \\
\text { gallinarum }\end{array}$ \\
\hline 216 & Vitex negundo Linn. & Verbenaceae & Senuwar & Shurbs & $\begin{array}{l}\text { Leaves, } \\
\text { Fruits }\end{array}$ & $\begin{array}{l}\text { Skin disease, } \\
\text { rheumatoid. }\end{array}$ \\
\hline 217 & Vitis venifra Linn. & Vitaceae & Angur & Climber & Fruits & $\begin{array}{l}\text { Laxative, } \\
\text { stomachic, }\end{array}$ \\
\hline 218 & Withania somni fera Dunal. & Solanaceae & Ashwagandha & Herb & Roots & $\begin{array}{l}\text { Rheumatism, } \\
\text { female, disorders }\end{array}$ \\
\hline 219 & Xanthium spinosum Linn. & Asteraceae & Lapta & Herb & Buds & Diarrhoea \\
\hline 220 & Yucca gloriosa Linn. & Agavaceae & - & Shurbs & Fruits & $\begin{array}{l}\text { Purgative, } \\
\text { rheumatism, }\end{array}$ \\
\hline 221 & Ziziphus jujuba Mill. & Rhamnaceae & Ber & Shurbs & Fruits & $\begin{array}{l}\text { Tonic, antidot, } \\
\text { sedative. }\end{array}$ \\
\hline
\end{tabular}

These 221 documented plant species are spread over Dicotyledonous 186 species (84.16\%), Monocotyledons 32 species (14.48\%), Gymnosperms 1 species (0.45\%) and Pteridophytes 2 species $(0.90 \%)$ (Table-2). Habitually the investigated taxa fall under 6 groups - trees (43 in number ), shrubs ( 45 in number), Herbs (106 in number), Climbers ( 25 in number ), Epiphytes (01 in number) \& parasite (01 in number ), and their respective percentage has been defined (figure-1). 

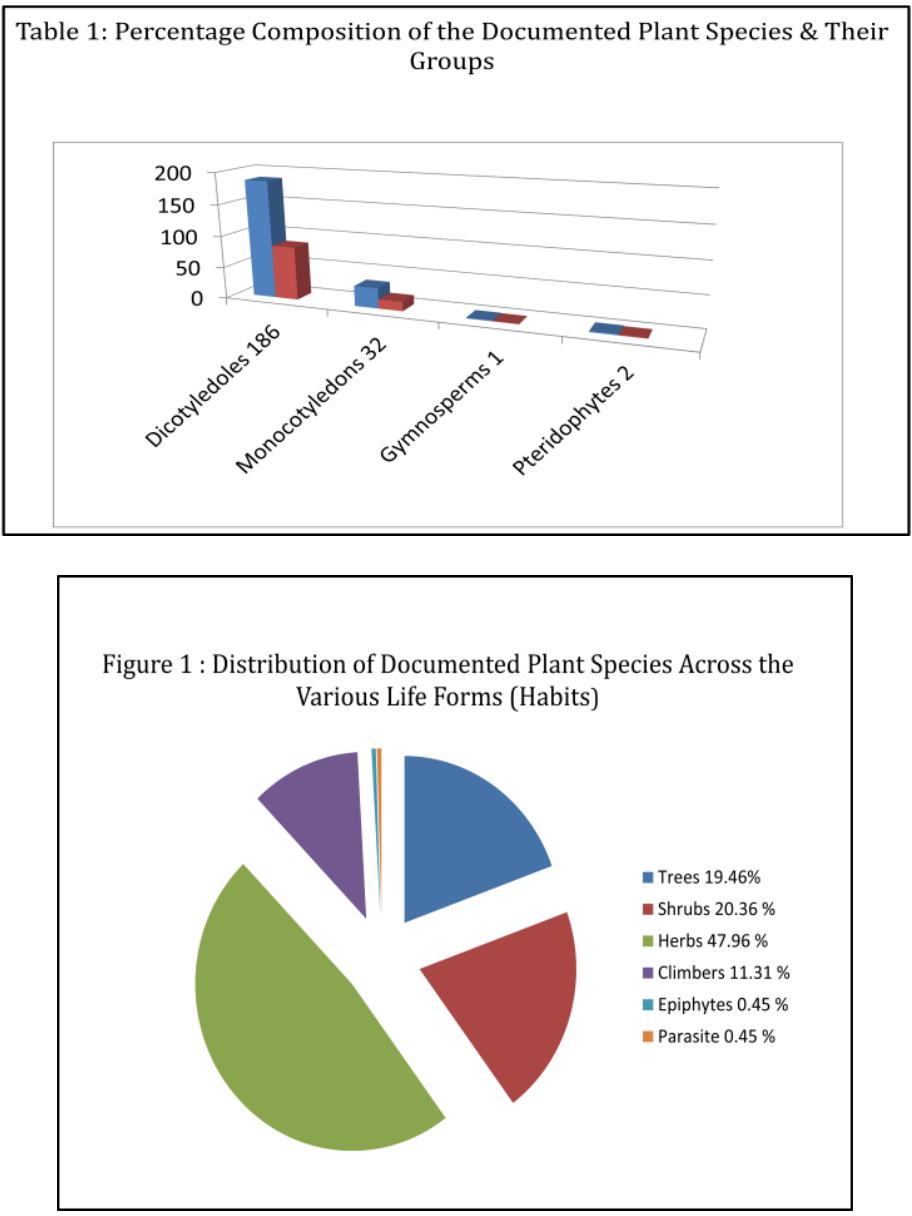

Distribution of medicinal plants Acording to family in this region

\begin{tabular}{|l|l|l|}
\hline Sn & family & no of sps \\
\hline 1. & Fabaceae & 35 \\
\hline 2. & Solanaceae & 21 \\
\hline 3 & Cucurbitacea & 30 \\
\hline 4. & Asteraceae & 19 \\
\hline 5 & Caesalpinaceae & 18 \\
\hline 6 & malvaceae,Moraceae,Apocynaceae & 17 \\
\hline 7 & Mimoceae,verbinaceae,Euphorbiceae & 16 \\
\hline 8 & Rutaceae, Apiaceae,Liliaceae, Poaceae & 15 \\
\hline 9. & $\begin{array}{l}\text { Amaranthaceae,chenopodiaceae, Myrtaceae, Zingerberaceae, } \\
\text { convulvulaceae, }\end{array}$ & 08 \\
\hline 10. & Acanthaceae, Annonaceae, Aracaceae,Brasicaeae & 7 \\
\hline
\end{tabular}

It has been observed that most of the Ethno Medicinal herbs are grown as Weeds, Climbers are as agricultural proposes, shrubs and trees are for ornamental, shade and timber proposes. Only very few plants are grown as purely medicinal proposes.

If awareness creation and motivation in rural and urban population towards Ethno medicinal Plants will be created to cultivate Ethno medicinal Plants in commercial Proposes as well as their personal needs. This will leads to upliftment of communities in rural as well as urban area of the district. It Will also ensure conservation of biodiversity.

\section{Acknowledgements}

Authour is thankful to Prof.(Dr) Rajeeva Bimal, Professor of Botany, BRA Bihar university, muzafarpur for Providing inspiration and all the authors from whose research and publications they have taken information on Ethno medicinal Plants. 


\section{References}

[1]. Ahmad Aftab and Rita Rani Sinha, Study of some indigenous medicinal Plants of Patna used to cure Different Gynecological ailments. Int.J.Mendel vol. 26(1-4),9-10,2009.

[2]. Ahmed Aftab and Hena Perween, Study of Medicinal Plants used in the Treatment of hypertension. Int.J. Mendel. Vol.26(1-4), 47.48,2009.

[3]. Anand Ratnesh Kumar \& Shailja Srivastava, India's opportunity for cultivation of Medicinal plants in Himalaya Region of Bihar (Champaran), India , Recent Advances in Medicinal Plants and their cultivahan , Edrs Aloka KUmari, R.K .Lal, K. Khajurai, Manglam Publications Delhi PP-191-209,2012.

[4]. Annonymous, The useful Plants of India (2006),NISCAIR-CSIR, New Delhi.

[5]. Bedi,Ramesh, jangal Ki jadi - Butiyan, Raj kamal Prakashan, New Delhi, Patna.

[6]. Bedi, Ramesh, Manaw upyogi Ped, Raj kamal Prakashan New Delhi, Patna.

[7]. Devraj, T.L. 1985, Ayurvedic remedies for common diseases, sterling Publisher Private Ltd, New Delhi .

[8]. Jain, S.K.,Medicinal Plants (2011), National Book Trust, INDIA,New Delhi.

[9]. Kritkar,K.R. and Basu, B.B. 1935 Indian Medicinal Plants, Vol.1-III, Lalit Mohan Basu, Allahabad, PP -30-120,142,160.

[10]. Kunul , kandir, Studies of herbal Plants used to Promote hemoglobin in blood in Jharkhand, Biospectra : vol.6(2), sept.(spl.issue)2011,PP,227-230.

[11]. Ojha, Neerjan \& Sudansha kumar, Documentation of some wild flowers used as food supplement by the tribal and others in Jharkhand, Biospeetra Vol 6 (2), sept. (spl.Issue) 2011,PP 177-180.

[12]. Pareek,Aparne and P.C Trivedi, Indigenous Knowledge and Plant Genetic Resource conservation in Kaladera Region, Jaipur, Int. J. mendel, vol.27(1-2),43-44,2010.

[13]. Prasad, V. Ethnomedicinal Plants of Birgunj, Nepal, Int. J. Mendel, vol.26(1-4),11-12,2009

[14]. Singh,Basant Narain, Study of Medicinal Plants of Gopalganj used in the treatment of cardiovascular disease, Biochemical science. Vol-1. Issue 3, July 2011, PP-47-48

[15]. Singh,Basant Narain \& Pankaj Kumar, Cultivation of medicinal Plants for poverty Alleviation in context of Bihar, Recent Advances in Medicinal Plants and their Caltivation, Edrs. Aloka Kumari Lal, R.K. R.K. Khajuria, Manglam Publications Delhi (India), PP $110-128,2012$

[16]. Sinha , K.R.1996. Ethnobotany. The renaissance of traditional medicine.

[17]. Sinha,Satish Kumar \& Ramesh Chandra, Medico-Ethnobotanical Study of Madhubani district Bihar, Biospectra: vol, 6 (1), March 2011 P.P 131-134.

[18]. Singh , R.S, 1998. Paschatya Dravya Guna vigyan. 5 vols. Motilal Banarsi Das, New Delhi 